\title{
Avaliação laboratorial do uso de solução salina hipertônica e isotônica e de furosemida no tratamento da intoxicação por amônia em bovinos
}

\author{
Laboratorial evaluation of hypertonic and isotonic saline solution and furosemide use for the ammonia \\ poisoning treatment in cattle
}

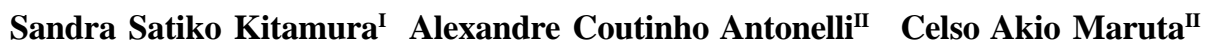 \\ Maria Claudia Araripe Sucupira ${ }^{\text {II }}$ Clara Satsuki MoriI ${ }^{I}$ Letícia Andreza Yonezawa ${ }^{\text {III }}$ \\ Lilian Emy dos Santos Michima ${ }^{\mathrm{II}}$ Pierre Castro Soares ${ }^{\mathrm{II}}$ Enrico Lippi Ortolani ${ }^{\mathrm{II}}$
}

\section{RESUMO}

Para testar a eficiência de vários tratamentos de intoxicação por amônia em bovinos, foram utilizados 25 garrotes que receberam cloreto de amônio por infusão intravenosa (iv) até o surgimento de quadro convulsivo. Em seguida, os animais foram alocados em um dos cinco grupos experimentais e tratados da seguinte forma: 1) controle: infusão (iv) de $300 \mathrm{~mL}$ de solução salina isotônica (SSI), no decorrer de $4 \mathrm{~h}$; 2) infusão (iv) de $30 \mathrm{~mL} \mathrm{~kg}^{-1} \mathrm{PV}$ de SSI no decorrer de 4 h e administração de $4 L$ de água intraruminal por meio de sonda esofágica (ASE); 3) mesmo tratamento do grupo 2 e dose única (iv) de furosemida $\left(2 \mathrm{mg} \mathrm{kg}^{-1} \mathrm{PV}\right)$ (F); 4) injeção (iv) de $5 \mathrm{~mL} \mathrm{~kg}^{-1} \mathrm{PV}$ de solução salina hipertônica (SSH) 7,2\% nos primeiros 30min, seguida de $20 \mathrm{~mL} \mathrm{~kg}^{-1} \mathrm{PV}$ de SSI e $4 \mathrm{~L}$ de ASE; 5) mesmo tratamento do grupo 4 e dose única de F. No decorrer de 4 h após a convulsão, foram determinados os teores plasmáticos de amônia e glicose, ureia, creatinina, potássio e sódio séricos, volume e gravidade específica da urina, e excreção urinária de amônio e ureia. No momento da convulsão, os teores de amônia plasmáticos foram muito altos e idênticos em todos os tratamentos, mas no $120^{\circ} \mathrm{min}$, nos grupos tratados com associação de SSH+SSI+ASE (grupos 4 e 5), houve diminuição desse metabólito. O uso de furosemida (grupos 3 e 5) não aumentou a excreção total de urina. A terapia com associação de SSH+SSI+ASE aumentou ainda o volume urinário e a excreção percentual urinária de ureia e amônia durante o período crítico da $1^{a}$ hora de tratamento, mas o uso de SSI+ASE (grupos 2 e 3) teve resultados intermediários. A eficiência do tratamento com SSH+SSI+ASE ou SSI+ASE foi superior ao grupo controle. Embora com efeito menor que o observado com $S S H+S S I+A S E$, a SSI+ASE promoveu melhora no quadro clínico geral e, ao término do experimento, promoveu também uma adequada desintoxicação da amônia.

Palavras-chave: intoxicação por amônia, tratamento, hipertônica, furosemida, bovinos.

\section{ABSTRACT}

To test the efficiency of some treatments for ammonia poisoning in cattle, 25 steers were used. Ammonium chloride solution was infused intravenously (iv) in each steer until the onset of convulsive episode. Thereafter, the animals were distributed in one of the five different groups, as follows: 1) control: infusion (iv) of $300 \mathrm{~mL}$ isotonic saline solution (ISS) throughout the following $4 \mathrm{~h} ; 2$ ) infusion (iv) of ISS $30 \mathrm{~mL} \mathrm{~kg}^{-1} \mathrm{BW}$ throughout the following $4 \mathrm{~h}$ and administration of $4 L$ water (W) through stomach tube; 3) the same as group 2 , plus a single dose (iv) $2 g$ furosemide $\mathrm{kg}^{-1} \mathrm{BW}(\mathrm{F})$; 4) injection of $5 \mathrm{~mL} \mathrm{kg^{-1 }} \mathrm{BW}$ hypertonic saline solution (HSS) (7.2\%) (iv) throughout the first 30min, followed by $20 \mathrm{~mL} \mathrm{~kg}$ $B W$ of ISS and $4 L \mathrm{~W}$.; 5) the same as group 4 and a single dose of F. For the next $4 \mathrm{~h}$ after the convulsion, plasma concentration of ammonia and glucose, serum urea, creatinine, sodium and potassium, urine total volume and specific gravity, and urinary excretion of ammonium and urea were followed. At the convulsion, the ammonia blood levels were high and similar among the groups, but at the $120^{\text {th }}$ min the animals treated with HSS $+I S S+W$ (groups 4 and 5) had lower values than the control. Furosemide (groups 3 and 5) did not prevent an increase in the total excretion of urine. The therapy with HSS $+I S S+W$ still increased the urinary volume and the total urinary excretion of urea and ammonium

'Universidade Anhembi-Morumbi. Rua Dr. Luiz Migliano, 871, ap. 62C, 05711-001, São Paulo, SP, Brasil. E-mail: sandrakitamura@gmail.com. Autor para correspondência.

IIDepartamento de Clínica Médica (VCM), Faculdade de Medicina Veterinária e Zootecnia (FMVZ), Universidade de São Paulo (USP), São Paulo, SP, Brasil.

IIIFaculdade de Medicina Veterinária e Zootecnia (FMVZ), Universidade Estadual Paulista (Unesp), Campus de Botucatu, Botucatu, SP, Brasil. 
during the critical $1^{\text {st }} h$ of treatment, while the use of ISS $+W$ had intermediate results. The efficiency of the treatment with HSS $+I S S+W$ or ISS $+W$ was better than the control group. Although with lower efficiency as seen in the treatment with $H S S+I S S+W$, the ISS $+W$ caused an improvement in the clinical picture and at the end of the experiment generated an adequate ammonia detoxification.

Key words: ammonia intoxication, treatment, hypertonic, furosemide, cattle.

\section{INTRODUÇÃO}

A carência de proteína nos ruminantes geralmente ocorre no período de estiagem, sendo o mais limitante nutriente para o crescimento de bovinos criados em condições extensivas (MINSON, 1990; BORTOLUSSI et al., 1996). Para prevenir tal carência, é comumente adicionada a ureia ao sal mineralizado, como fonte muito rica em nitrogênio não proteico, para substituir parte significativa da proteína vegetal (OLIVEIRA, 1985). Embora o uso de ureia proporcione várias vantagens na alimentação de bovinos, a sua utilização inadequada pode acarretar sérios danos à saúde, podendo levar à morte, principalmente por intoxicação aguda (MORRIS \& PAYNE, 1970). Ainda que muitos utilizem a denominação errônea de intoxicação por ureia, o que se constata é que os animais sucumbem devido à hidrólise ruminal da ureia, gerando um acúmulo de amônia, a qual, se absorvida em grande quantidade, pode desencadear um grave quadro tóxico (HALIBURTON \& MORGAN, 1989).

Embora os efeitos do excesso de amônia sejam conhecidos, pouco ainda se tem avançado em tratamentos que antagonizem, com eficiência, os quadros súbitos e letais de intoxicação (ORTOLANI \& ANTONELLI, 2004). Até o momento, o tratamento usual é a rápida administração de ácidos fracos (ácido acético ou vinagre), transformando a amônia em amônio e diminuindo sua absorção pela parede ruminal (BARTLEY et al., 1976). ORTOLANI et al. (2000) foram os primeiros a obterem resultados amplamente satisfatórios quando, além do vinagre, instituíram um tratamento parenteral à base de hidratante isotônico e diurético de "alça" (furosemida). Posteriormente, KITAMURA (2002) recomendou a utilização dos aminoácidos do ciclo da ureia e de diurético no tratamento dos animais intoxicados, visando à maior diurese, ao aumento na excreção de quantidades de amônia ( $35 \%$ do cloreto de amônia infundido) e consequente à diminuição nos teores de amônia plasmática.

Outra possibilidade seria o uso intravenoso inicial de solução salina hipertônica, a qual rapidamente aumentaria a osmolaridade intravascular fazendo com que parte dos fluidos presentes no espaço extracelular, intraruminal e até mesmo intracelular migrem para os vasos sanguíneos, corrigindo rapidamente a hipovolemia e o fluxo sanguíneo renal e aumentando assim a produção urinária (CONSTABLE, 1999). Para garantir a eficiência desse tratamento faz-se necessária a administração posterior de fluidos isotônicos por via intravenosa, oral ou intraruminal para permitir que esses fluidos possam migrar para o espaço intravascular por diferença de gradiente osmótico. Uma terceira possibilidade de tratamento seria aumentar a excreção renal de amônio com o uso de diurético de ação na alça de Henle. A furosemida foi utilizada com sucesso por KITAMURA (2002), o qual observou que tal diurético aumentou o volume urinário e diminuiu o edema pulmonar em bovinos e ratos com intoxicação experimental por amônia. Embora essas alternativas possam ter uma ação benéfica no tratamento da intoxicação superaguda por amônia, há necessidade de se realizar uma avaliação experimental utilizando tratamentos que possam reduzir as concentrações plasmáticas de amônia e seus efeitos deletérios ao organismo animal.

Portanto, o presente estudo objetivou avaliar a eficiência de tratamentos em quadro de intoxicação superaguda experimental em bovinos por amônia, por meio de infusão de solução salina isotônica ou hipertônica, associada à administração oral de água, e de uso ou não de furosemida, sendo acompanhadas variáveis laboratoriais que indiquem o grau de recuperação e a capacidade de desintoxicação daquela substância.

\section{MATERIAL E MÉTODOS}

Foram utilizados 25 garrotes hígidos, mestiços, com cerca de 18 meses de idade e $250 \mathrm{~kg}$ de peso vivo. Os animais foram mantidos confinados, em baias individuais, nas dependências do "Galpão de Experimentação Clínica de Ruminantes" da Faculdade de Medicina Veterinária e Zootecnia (FMVZ) da Universidade de São Paulo (USP). Receberam dieta composta por feno de coast cross (Cynodon dactilon (L) pers), oferecido ad libitum, e $0,5 \%$ de seus pesos vivos em concentrados, a fím de que a ração não apresentasse mais de $12 \%$ de proteína bruta, nem qualquer fonte de nitrogênio não protéico.

A intoxicação foi realizada somente uma vez em cada animal no decorrer do período experimental (KITAMURA et al., 2003), por meio de infusão intravenosa (iv) de $1,5 \mathrm{~mol} \mathrm{~L} \mathrm{~L}^{-1}$ de cloreto de amônio diluído em água bidestilada, livre de outros compostos 
nitrogenados, estabilizada num pH 7,0. A infusão foi realizada por cateter de polietileno de $30 \mathrm{~cm} \times 1,4 \mathrm{~mm}$ fixado na veia jugular esquerda. Foram injetados $400 \mathrm{~mL}$ $\mathrm{h}^{-1}$, sendo utilizado um controlador automático de fluxo (DigiBomb $^{\circledR}$, São Paulo). Após o surgimento do primeiro episódio convulsivo, a infusão era interrompida e de imediato era iniciado o tratamento. Os animais foram distribuídos aleatoriamente em um dos cinco tratamentos, e cada grupo continha cinco bovinos, num delineamento multifatorial:

- Grupo 1 (G1) - controle: infusão (iv) de $300 \mathrm{~mL}$ de solução salina isotônica (SSI) no decorrer de $4 \mathrm{~h}$;

- Grupo 2 (G2) - tratado com $30 \mathrm{~mL} \mathrm{~kg}^{-1} \mathrm{PV}$ de SSI (iv) no decorrer de $4 \mathrm{~h}$, associado à administração de 4L de água intrarruminal por meio de sonda esofágica (ASE);

- Grupo 3 (G3) - mesmo tratamento do G2 e dose única (iv) de furosemida $\left(2 \mathrm{mg} \mathrm{kg}^{-1} \mathrm{PV}\right)$;

- Grupo 4 (G4) - tratado inicialmente com $5 \mathrm{~mL} \mathrm{~kg}^{-1} \mathrm{PV}$ de solução salina hipertônica (SSH) 7,2\% $\left(2460,7 \mathrm{mOsm} \mathrm{L}^{-1}\right)$ infundida nos primeiros $30 \mathrm{~min}$, seguida de $20 \mathrm{~mL} \mathrm{~kg}^{-1}$ PV de SSI (iv), aplicada no decorrer de $3 \mathrm{~h}$ e $30 \mathrm{~min}$ subsequentes, eassociada a administração de 4L de ASE;

- Grupo 5 (G5) - mesmo tratamento do grupo 4 e dose única (iv) de furosemida $\left(2 \mathrm{mg} \mathrm{kg}^{-1} \mathrm{PV}\right.$ ).

Foram coletadas amostras de sangue no início do experimento após surgimento do episódio convulsivo (momento zero) e, no decorrer das $4 \mathrm{~h}$ de tratamento, para a determinação de amônia e glicose plasmáticas $\left(\right.$ Raichem $^{\circledR}$, EUA; Biotrol $^{\circledR}$, França) e ureia (segundo TALKE \& SCHUBERT, 1964), creatinina (segundo LUTSGARTEN \& WENK, 1972), sódio e potássio séricos (fotometria de chama). Coletou-se também toda a urina durante o experimento para determinar o volume, a gravidade específica da urina por refratometria e as concentrações de amônio e ureia em alíquotas, sendo os valores multiplicados ao volume correspondente para se saber a concentração de amônio ou ureia urinária total.

Dependendo da distribuição dos dados, analisados pelo teste de Kolgomorov e Smirnov, estes foram avaliados segundo métodos estatísticos paramétricos ou nãoparamétricos. Na ocorrência do primeiro caso, os dados foram inicialmente avaliados por meio de teste $\mathrm{F}$ (análise de variância) e, quando significativo, as médias foram comparadas pelo teste de múltipla amplitude de Tukey (SAMPAIO, 1998). No caso de testes não paramétricos, estes foram analisados pelo teste de Kruskal-Wallis (SIEGEL, 1975). As análises foram realizadas com o auxílio do programa estatístico computadorizado (MINITAB
13.0, EUA), considerando diferença significativa quando $\mathrm{P}<0,05$.

\section{RESULTADOS E DISCUSSÃO}

Os teores de amônia plasmática no momento zero foram máximos (Tabela 1), mas não foram diferentes entre os cinco grupos e foram diminuindo gradativamente. Embora existisse uma expectativa de ocorrer uma diminuição mais drástica e rápida nessas concentrações de amônia, nos grupos tratados, esta só veio ocorrer mais tardiamente (120min) no G4 e no G5, medicados com solução hipertônica de cloreto de sódio, seguida dos demais tratamentos, que passaram a se diferenciar do grupo controle, a partir do $180^{\circ} \mathrm{min}$ (Tabela 1). Mesmo considerando que os teores de amônia plasmáticos não tivessem apresentado uma queda mais pronunciada, os tratamentos de alguma forma provocaram uma melhora no quadro geral, demonstrada pelo quadro clínico (diminuição da frequência cardíaca, respiratória e temperatura retal, reversão do edema pulmonar e retorno do apetite e da posição quadrupedal) e pelas variáveis laboratoriais.

Os dados totais de volume urinário indicaram que os tratamentos com fluidos associados ou não ao uso de diurético (G2: $127,5 \mathrm{~mL} \mathrm{~kg}^{-1} \mathrm{PV}^{0,75}$; G3: 208,3mL kg-1 $\mathrm{PV}^{0,75}$; G4: 216,8mL kg-1 $\mathrm{PV}^{0,75}$; G5: $\left.231,5 \mathrm{~mL} \mathrm{~kg}^{-1} \mathrm{PV}^{0,75}\right)$ proporcionaram uma maior $(\mathrm{P}<0,03)$ excreção urinária em relação ao grupo controle $(\mathrm{G} 1$ : $90,3 \mathrm{~mL} \mathrm{~kg}^{-1} \mathrm{PV}^{0,75}$ ). A maior eliminação de urina é um ponto fundamental para a recuperação dos bovinos intoxicados, visto que foi constatada uma correlação positiva entre o volume urinário e a quantidade de amônio eliminado (KITAMURA, 2002; SANTOS, 2005). Estudos anteriores demonstraram que a solução salina hipertônica, além de aumentar o volume plasmático em até $20 \%$, provocou concomitantemente um incremento na excreção urinária devido a um acréscimo na taxa de filtração glomerular, em especial durante a primeira meia hora após o tratamento (CONSTABLE et al., 1994; ROEDER et al., 1997). Neste estudo não foi determinada a taxa de filtração glomerular, mas é possível que isso tenha ocorrido, visto que o volume urinário eliminado foi muito maior em animais tratados com $\mathrm{SSH}+\mathrm{SSI}+\mathrm{AES}$, em comparação com os demais tratamentos.

O valor total da gravidade específica urinária foi maior $(\mathrm{P}<0,02)$ nos animais do grupo controle (G1: 1,008 $\pm 0,003)$ que nos animais do grupo 2 $(1,006 \pm 0,002)$ e grupo $3(1,006 \pm 0,004)$. Isso pode ser uma consequência do menor volume urinário excretado no decorrer do período de tratamento no grupo controle, tornando menos diluídos os metabólitos 
Tabela 1 - Teores de amônia $\left(\mu \mathrm{mol} \mathrm{L} \mathrm{L}^{-1}\right)$, glicose plasmática $\left(m m o l \mathrm{~L}^{-1}\right)$ e sódio sérico $\left(m m o l \mathrm{~L}^{-1}\right)$ em animais submetidos à intoxicação por amônia e a diferentes protocolos de tratamento (Grupos 1 a 5 ).

\begin{tabular}{|c|c|c|c|c|c|c|c|c|c|}
\hline & 0 & 10 & 20 & 30 & 60 & 90 & 120 & 180 & 240 \\
\hline \multirow[b]{2}{*}{ G1 } & $1806+$ & $1452+$ & $1440+$ & $1291+$ & $1029+$ & $747+$ & $617+$ & $402+$ & $230+$ \\
\hline & $462^{\mathrm{Aa}}$ & $428^{\mathrm{Aa}}$ & $555^{\text {Aab }}$ & $542^{\mathrm{Aab}}$ & $474^{\mathrm{Abc}}$ & $330^{\text {Acd }}$ & $343^{\mathrm{Ad}}$ & $246^{\text {Ad }}$ & $156^{\mathrm{Ad}}$ \\
\hline \multirow{3}{*}{ G2 } & $1544+$ & $1287+$ & $1169+$ & $900 \pm$ & $565+$ & $358+$ & $220+$ & $68+$ & $52+$ \\
\hline & 107ד & 12011 & 170 & A & Abc & J & $\mathrm{ABcd}$ & $\mathrm{Bd}$ & Bd \\
\hline & & & & & $210^{\mathrm{ADC}}$ & & $97^{\mathrm{ABCO}}$ & & $6^{\mathrm{Ba}}$ \\
\hline \multirow{2}{*}{ G3 } & $2216 \pm$ & $1555 \pm$ & $1266 \pm$ & $1038 \pm$ & $654 \pm$ & $456 \pm$ & $368 \pm$ & $157 \pm$ & $89 \pm$ \\
\hline & $88^{\mathrm{Aa}}$ & $706^{\mathrm{Aa}}$ & $595^{\mathrm{Aa}}$ & $528^{\mathrm{Aab}}$ & $420^{\mathrm{Abc}}$ & $282^{\text {Acd }}$ & $172^{\mathrm{ABcd}}$ & $113^{\mathrm{ABd}}$ & $18^{\mathrm{Abd}}$ \\
\hline \multirow{2}{*}{ G4 } & $1505 \pm$ & $1218 \pm$ & $1069 \pm$ & $984 \pm$ & $714 \pm$ & $473 \pm$ & $214 \pm$ & $73 \pm$ & $71 \pm$ \\
\hline & $464^{\mathrm{Aa}}$ & $311^{\mathrm{Aa}}$ & $286^{\text {Aab }}$ & $282^{\text {Aab }}$ & $248^{\mathrm{Abc}}$ & $181^{\text {Acd }}$ & $79^{\mathrm{Bd}}$ & $31^{\mathrm{Bd}}$ & $57^{\mathrm{Bd}}$ \\
\hline \multirow{2}{*}{ G5 } & 1841 & $1683 \pm$ & $1301 \pm$ & $1132 \pm$ & $713 \pm$ & $453 \pm$ & $214 \pm$ & $84 \pm$ & $59 \pm$ \\
\hline & $\pm 458^{\mathrm{Aa}}$ & $476^{\mathrm{Aa}}$ & $504^{\text {Aab }}$ & $475^{\text {Aab }}$ & $308^{\mathrm{Abc}}$ & $279^{\text {Acd }}$ & $48^{\mathrm{Bcd}}$ & $30^{\mathrm{Bd}}$ & $20^{\mathrm{Bd}}$ \\
\hline \multirow{2}{*}{ G1 } & $12,0 \pm$ & $13,7 \pm$ & $14,6 \pm$ & $14,5 \pm$ & $14,4 \pm$ & $13,6 \pm$ & $12,8 \pm$ & $11,7 \pm$ & $12,3 \pm$ \\
\hline & $3,6^{\mathrm{Aa}}$ & $3,7^{\mathrm{Aa}}$ & $3,9^{\mathrm{Aa}}$ & $4,3^{\mathrm{Aa}}$ & $4,8^{\mathrm{Aa}}$ & $4,5^{\mathrm{Aa}}$ & $4,0^{\mathrm{Aa}}$ & $1,8^{\mathrm{Aa}}$ & $1,5^{\mathrm{Aa}}$ \\
\hline \multirow{2}{*}{ G2 } & $10,2 \pm$ & $13,3 \pm$ & $14,6 \pm$ & $14,9 \pm$ & $13,8 \pm$ & $12,5 \pm$ & $11,7 \pm$ & $9,3 \pm$ & $8,2 \pm$ \\
\hline & $3,5^{\text {Aabc }}$ & $3,1^{\mathrm{abc}}$ & $3,2^{\mathrm{Aa}}$ & $3,2^{\mathrm{Aa}}$ & $3,8^{\mathrm{Aab}}$ & $3,8^{\text {Aabc }}$ & $4,6^{\text {Aabc }}$ & $3,7^{\mathrm{Abc}}$ & $2,9^{\mathrm{Bc}}$ \\
\hline \multirow{2}{*}{ G3 } & $11,3 \pm$ & $13,8 \pm$ & $14,1 \pm$ & $14,1 \pm$ & $13,2 \pm$ & $12,3 \pm$ & $11,7 \pm$ & $9,9 \pm$ & $8,3 \pm$ \\
\hline & $3,6^{\mathrm{Aa}}$ & $3,3^{\mathrm{Aa}}$ & $4,0^{\mathrm{Aa}}$ & $3,6^{\mathrm{Aa}}$ & $3,3^{\mathrm{Aa}}$ & $2,9^{\mathrm{Aa}}$ & $2,8^{\mathrm{Aa}}$ & $2,5^{\mathrm{Aa}}$ & $2,4^{\mathrm{Ba}}$ \\
\hline \multirow{2}{*}{ G4 } & $10,5 \pm$ & $11,8 \pm$ & $12,2 \pm$ & $12,4 \pm$ & $12,6 \pm$ & $11,3 \pm$ & $9,9 \pm$ & $8,3 \pm$ & $7,0 \pm$ \\
\hline & $2,8^{\mathrm{Aab}}$ & $21,9^{\mathrm{Aa}}$ & $1,5^{\mathrm{Aa}}$ & $2,3^{\mathrm{Aa}}$ & $3,2^{\mathrm{Aa}}$ & $2,1^{\mathrm{Aa}}$ & $1,8^{\text {Aabc }}$ & $1,6^{\mathrm{Abc}}$ & $1,6^{\mathrm{Bc}}$ \\
\hline \multirow{2}{*}{ G5 } & $9,1 \pm$ & $11,0 \pm$ & $10,6 \pm$ & $10,8 \pm$ & $11,0 \pm$ & $10,8 \pm$ & $9,5 \pm$ & $8,2 \pm$ & $7,1 \pm$ \\
\hline & $2,8^{\mathrm{Aa}}$ & $2,1^{\mathrm{Aa}}$ & $2,9^{\mathrm{Aa}}$ & $3,1^{\mathrm{Aa}}$ & $2,7^{\mathrm{Aa}}$ & $2,2^{\mathrm{Aa}}$ & $2,0^{\mathrm{Aa}}$ & $2,3^{\mathrm{Aa}}$ & $3,1^{\mathrm{Ba}}$ \\
\hline \multirow{2}{*}{ G1 } & $140,8 \pm$ & $139,4 \pm$ & $139,4 \pm$ & $138,5 \pm$ & $139,5 \pm$ & $139,0 \pm$ & $139,6 \pm$ & $139,2 \pm$ & $138,7 \pm$ \\
\hline & $3,2^{\mathrm{Aa}}$ & $3,0^{\mathrm{Ba}}$ & $3,2^{\mathrm{Ba}}$ & $5,4^{\mathrm{Ba}}$ & $4,0^{\mathrm{Ba}}$ & $3,6^{\mathrm{Ba}}$ & $3,9^{\mathrm{Ba}}$ & $3,2^{\mathrm{ABa}}$ & $2,5^{\mathrm{ABa}}$ \\
\hline \multirow{2}{*}{ G2 } & $138,7 \pm$ & $139,1 \pm$ & $138,9 \pm$ & $139,5 \pm$ & $138,8 \pm$ & $139,5 \pm$ & $139,1 \pm$ & $139,8 \pm$ & $140,2 \pm$ \\
\hline & $3,2^{\mathrm{Aa}}$ & $3,8^{\mathrm{Ba}}$ & $3,7^{\mathrm{Ba}}$ & $3,6^{\mathrm{Ba}}$ & $3,1^{\mathrm{Ba}}$ & $3,2^{\mathrm{Ba}}$ & $3,4^{\mathrm{Ba}}$ & $3,5^{\mathrm{ABa}}$ & $3,1^{\mathrm{ABa}}$ \\
\hline \multirow{2}{*}{ G3 } & $139,5 \pm$ & $139,0 \pm$ & $139,1 \pm$ & $139,5 \pm$ & $138,5 \pm$ & $139,1 \pm$ & $137,6 \pm$ & $136,4 \pm$ & $136,9 \pm$ \\
\hline & $2,5^{\mathrm{Aa}}$ & $2,8^{\mathrm{Ba}}$ & $2,7^{\mathrm{Ba}}$ & $3,1^{\mathrm{Ba}}$ & $2,3^{\mathrm{Ba}}$ & $2,4^{\mathrm{Ba}}$ & $2,3^{\mathrm{Ba}}$ & $1,8^{\mathrm{Aa}}$ & $2,3^{\mathrm{Aa}}$ \\
\hline \multirow{2}{*}{ G4 } & $137,8 \pm$ & $143,4 \pm$ & $148,6 \pm$ & $150,2 \pm$ & $149,0 \pm$ & $146,8 \pm$ & $146,5 \pm$ & $145,5 \pm$ & $145,1 \pm$ \\
\hline & $1,4^{\mathrm{Ad}}$ & $1,6^{\mathrm{Ac}}$ & $5,4^{\mathrm{Aab}}$ & $1,2^{\mathrm{Aa}}$ & $2,4^{\mathrm{Aab}}$ & $2,5^{\mathrm{Aabc}}$ & $2,1^{\text {Aabc }}$ & $1,8^{\mathrm{Abc}}$ & $1,8^{\mathrm{Abc}}$ \\
\hline \multirow{2}{*}{ G5 } & $140,9 \pm$ & $145,7 \pm$ & $152,8 \pm$ & $150,2 \pm$ & $149,0 \pm$ & $147,2 \pm$ & $147,0 \pm$ & $144,4 \pm$ & $143,5 \pm$ \\
\hline & $3,3^{\mathrm{Ae}}$ & $4,7^{\text {Acd }}$ & $3,9^{\mathrm{Aa}}$ & $2,3^{\text {Aab }}$ & $2,4^{\text {Aabc }}$ & $1,6^{\mathrm{Abcd}}$ & $1,9^{\mathrm{Abcd}}$ & $1,6^{\text {Ade }}$ & $1,1^{\text {Ade }}$ \\
\hline
\end{tabular}

Letras minúsculas distintas na mesma linha indicam diferença estatística entre momentos experimentais

Letras maiúsculas distintas na mesma coluna indicam diferença estatística entre grupos experimentais

excretados. Não existiu diferença significativa entre o grupo controle e os medicados com $\mathrm{SSH}+\mathrm{SSI}+\mathrm{ASE}$ ou $\mathrm{SSH}+\mathrm{SSI}+\mathrm{ASE}$ associado à furosemida (G4: $1,007 \pm 0,003$; G5: $1,007 \pm 0,001$ ), pois sabidamente a excreção de sódio e cloro pela urina é muito maior nos animais infundidos com solução hipertônica (ROEDER et al., 1997).

A infusão de solução saturada de cloreto de sódio promoveu no G4 e no G5 aumento significativo das concentrações de sódio sérico (Tabela 1), como previamente citado na literatura (CONSTABLE, 1999), porém estas se mantiveram dentro dos valores de referência $\left(132-152 \mathrm{mmol} \mathrm{L}^{-1}\right)$ para bovinos (KANEKO et al., 2008). Embora não tenham sido mensurados os teores totais de sódio na urina, provavelmente, essa normonatriemia foi mantida devido à grande capacidade de excreção de sódio pela urina em animais tratados com solução salina hipertônica (ROEDER et al.,1997; CONSTABLE, 1999).

Todos os animais no momento zero do trabalho apresentaram teores relativamente altos de potássio sérico (Tabela 2), mas ainda dentro dos valores físiológicos $\left(3,9\right.$ a $\left.5,8 \mathrm{mmol} \mathrm{L}^{-1}\right)$ (KANEKO et al., 2008). Essas médias iniciais diminuíram em todos os grupos. Essa hipercalemia pode estar relacionada à acidose metabólica, distúrbio comum em animais com 
Tabela 2 - Teores de potássio $\left(m m o l ~ L^{-1}\right.$ creatinina $\left(\mathrm{mmol} \mathrm{L}^{-1}\right)$ e ureia $\left(\mathrm{mmol} \mathrm{L}^{-1}\right)$ séricos em animais submetidos à intoxicação por amônia e a diferentes protocolos de tratamento (Grupos 1 a 5 ).

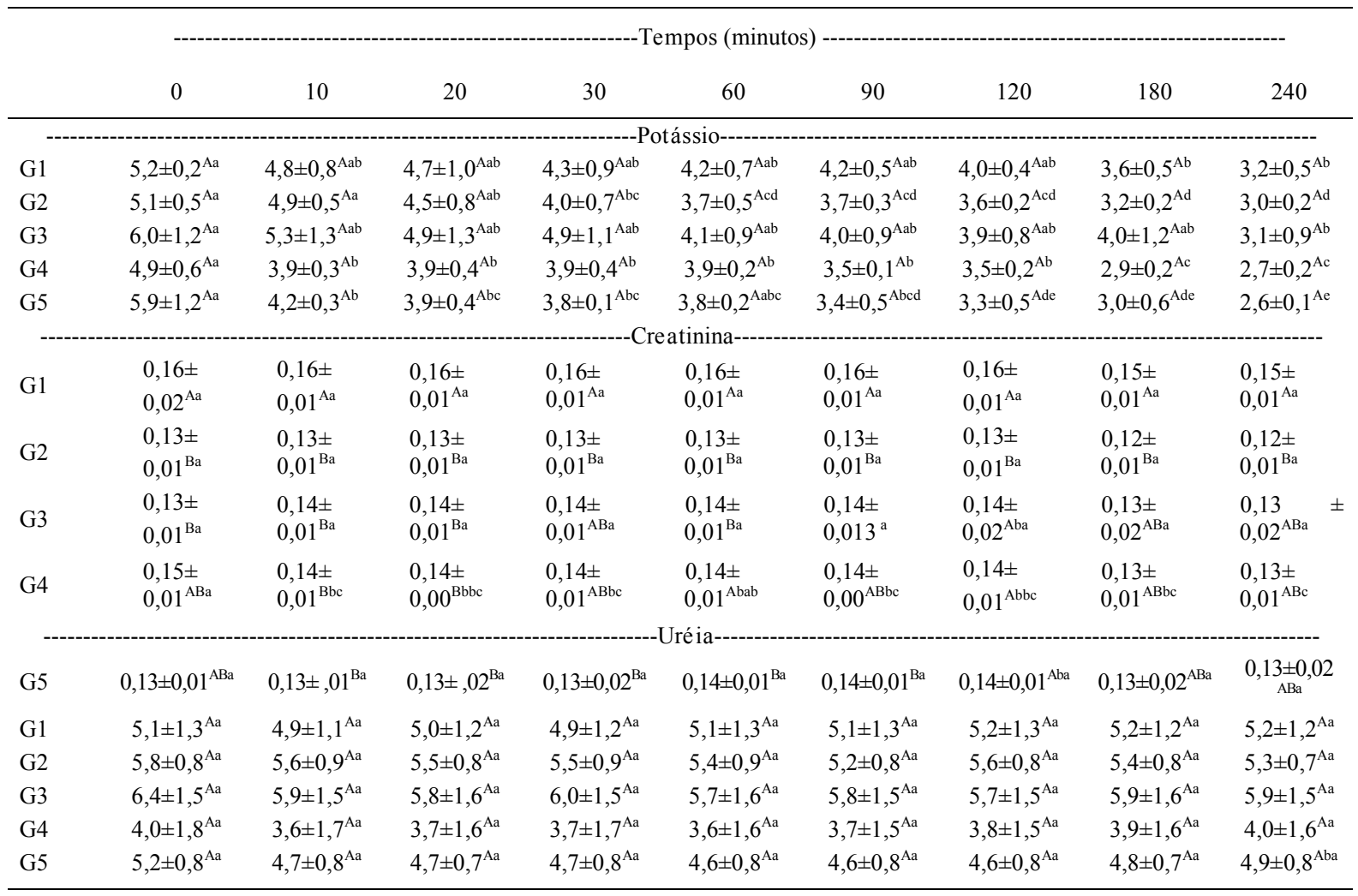

Letras minúsculas distintas na mesma linha indicam diferença estatística entre momentos experimentais.

Letras maiúsculas distintas na mesma coluna indicam diferença estatística entre grupos experimentais.

intoxicação por amônia (ORTOLANI \& ANTONELLI, 2004). Um dos mecanismos que o organismo utiliza para combater a acidose metabólica ocorre por meio da troca de íons $\mathrm{H}^{+}$da corrente circulatória pelo potássio do meio intracelular, provocando aumento desse íon na circulação sanguínea (ORTOLANI \& ANTONELLI, 2004; DIBARTOLA, 2007).

Um estado de hiperglicemia se desenvolveu durante todo o experimento nos animais dos cinco grupos (Tabela 1). Essa hiperglicemia é decorrente da interferência da hiperamoniemia sobre os hormônios que regulam o metabolismo da glicose, ou seja, quanto maior os teores desse composto nitrogenado, maior a liberação de glucagon, que estimula a gliconeogênese, e da adrenalina, que, por sua vez, estimula a glicogenólise, gerando uma redução no uso periférico da glicose por menor liberação de insulina (ORTOLANI \& ANTONELLI, 2004). É importante frisar que, no último momento (240min), os animais dos grupos indistintamente tratados apresentaram menores teores de glicose no plasma que o grupo controle, indicando a influência positiva destes.
Os maiores teores de creatinina séricos no decorrer do experimento indicaram que o grupo controle sempre esteve mais desidratado que os demais animais que foram medicados com associação de soluções hidratantes (Tabela 2). Não existiu diferença nos teores de ureia sérica, no decorrer do experimento entre ou dentro dos grupos (Tabela 2). Esse resultado deve ser analisado com ressalvas. Independente do tratamento, todos os animais apresentaram no período uma maior atividade do ciclo da ureia, visto que foram desafiados a transformar altas quantidades de amônia em ureia, o que elevaria os teores de ureia no sangue.

A confirmação de que todos os animais eliminaram significativas quantidades de ureia pela urina, no decorrer das $4 \mathrm{~h}$ de experimento, está apresentada na tabela 3. Segundo MARTIN JR. et al. (1981), no ciclo da ureia, um mol de amônia é transformado num mol de ureia, indicando a desintoxicação do primeiro composto. Assim sendo, mesmo no grupo controle, onde o processo foi menos pronunciado, a eliminação global de ureia urinária em $4 \mathrm{~h}$ foi de cerca de $62,4 \mathrm{mM}$ ( $30 \%$ de um total de $208 \mathrm{mM}$ 
Tabela 3 - Média do total de $\mathrm{NH}_{4}{ }^{+} \mathrm{Cl}$ injetado para obtenção de convulsão e mediana da excreção percentual urinária de amônio ou ureia, sendo ambos na $1^{\text {a }}$ h e durante as $4 \mathrm{~h}$ de tratamento em relação ao $\mathrm{NH}_{4}^{+} \mathrm{Cl}$ injetado.

\begin{tabular}{|c|c|c|c|c|c|c|c|}
\hline \multirow[b]{2}{*}{ Grupos } & \multirow[b]{2}{*}{$\begin{array}{l}\text { Total de amônio } \\
\text { injetado }(\mathrm{mM})\end{array}$} & \multicolumn{6}{|c|}{ Excreção (\%) urinário de amônio e ureia em relação ao $\mathrm{NH}_{4} \mathrm{Cl}$ injetado } \\
\hline & & Amônio (1h) & $\begin{array}{c}\text { Amônio } \\
\text { (Global - 4h) }\end{array}$ & Ureia (1h) & $\begin{array}{l}\text { Ureia (Global } \\
\quad-4 \mathrm{~h})\end{array}$ & $\begin{array}{c}\text { Total de } \\
\text { amônio + ureia } \\
\text { (1h) }\end{array}$ & $\begin{array}{c}\text { Total de } \\
\text { amônio + ureia } \\
(4 \mathrm{~h})\end{array}$ \\
\hline G1 & $208 \pm 73,7^{\mathrm{a}}$ & $1,6^{\mathrm{c}}$ & $12,9^{b}$ & $1,9^{\mathrm{c}}$ & $30,0^{b}$ & $3,5^{\mathrm{c}}$ & $42,9^{b}$ \\
\hline $\mathrm{G} 2$ & $159 \pm 41,6^{\mathrm{a}}$ & $10,0^{\mathrm{ab}}$ & $28,2^{\mathrm{a}}$ & $24,4^{b}$ & $68,2^{\mathrm{a}}$ & $34,4^{b}$ & $96,4^{\text {a }}$ \\
\hline G3 & $132 \pm 29,0^{\mathrm{a}}$ & $7,6^{\mathrm{b}}$ & $32,3^{\mathrm{a}}$ & $18,2^{\mathrm{b}}$ & $71,5^{\mathrm{a}}$ & $25,8^{\mathrm{b}}$ & $103,8^{\text {a }}$ \\
\hline G4 & $193 \pm 66,3^{\mathrm{a}}$ & $13,9^{\mathrm{a}}$ & $20,5^{\mathrm{a}}$ & $35,7^{\mathrm{a}}$ & $76,5^{\mathrm{a}}$ & $49,6^{\mathrm{a}}$ & $97,0^{\mathrm{a}}$ \\
\hline G5 & $145 \pm 26,9^{a}$ & $14,0^{\mathrm{a}}$ & $37,9^{\mathrm{a}}$ & $32,5^{\mathrm{a}}$ & $77,4^{\mathrm{a}}$ & $46,5^{a}$ & $115,3^{\mathrm{a}}$ \\
\hline Significância & $\mathrm{P}>0,2$ & $\mathrm{P}<0,04$ & $\mathrm{P}<0,03$ & $\mathrm{P}<0,03$ & $\mathrm{P}<0,05$ & $\mathrm{P}<0,02$ & $\mathrm{P}<0,05$ \\
\hline
\end{tabular}

Letras distintas nas colunas indicam diferenças significativas entre os grupos experimentais.

de amônia injetada), ou seja, cerca de 12 vezes maior que o teor de ureia num litro de sangue. Ao término de 4h de tratamento, os animais medicados com cada um dos tratamentos tiveram uma maior excreção de amônio e de ureia que os do grupo controle. Esse resultado se repetiu individualmente tanto para a excreção de ureia com de amônia na $1^{\text {a }}$ h, quanto para a excreção global no decorrer das $4 \mathrm{~h}$ de tratamento. Deve-se ressaltar o resultado da excreção de amônio e ureia durante a $1^{\mathrm{a} h}$, em que se definiu claramente que os grupos medicados com $\mathrm{SSH}+\mathrm{SSI}+\mathrm{ASE}$ ou $\mathrm{SSH}+\mathrm{SSI}+\mathrm{ASE}$ associados à furosemida (grupos 4 e 5) tiveram maiores percentagens de excreção que os tratados com solução isotônica (G1, G2 e G3). Essa 1 $1^{\mathrm{a} h}$ é fundamental para o sucesso do tratamento proposto. Em casos graves de intoxicação natural por amônia, se não houver uma adequada depuração desse composto nesse momento, ocorre a morte do animal cerca de 20 a 30 minutos após o início do quadro convulsivo.

Esses resultados demonstraram claramente a importância que a hidratação, por meio de solução isotônica e/ou hipertônica de cloreto de sódio associada à administração oral de água, tem na depuração da amônia pela urina. Segundo ROEDER et al. (1997), em ambos os tratamentos supracitados, existe um aumento no volume plasmático, o qual por consequência provoca, em seguida, um incremento no volume urinário. Segundo KITAMURA (2002), quanto maior o volume urinário, maior a excreção de amônio pela urina, fato observado nos animais hidratados com associação de diferentes soluções em relação aos do grupo controle.

\section{CONCLUSÃO}

Como a metodologia empregada foi adequada para promover uma intoxicação por amônia em todos os animais, é possível concluir que o uso de $\mathrm{SSH}+\mathrm{SSI}+\mathrm{ASE}$, associada ou não à furosemida, promove um rápido e marcante aumento do volume urinário global, eliminando assim com grande eficiência a amônia e a ureia por esse fluido. Entretanto, o uso de furosemida não interfere no volume excretado de urina. Embora com efeito menor que o observado com solução salina hipertônica, a infusão de fluido isotônico promove melhora no quadro clínico geral e adequada desintoxicação da amônia.

\section{COMITÊ DE ÉTICA}

Este estudo foi realizado de acordo com os princípios éticos na experimentação animal e aprovado pela Comissão de Bioética da FMVZ - USP, processo n. $713 / 2005$.

\section{REFERÊNCIAS}

BARTLEY, E.E. et al. Ammonia toxicity in cattle. I. Rumen and blood changes associated with toxicity and treatments methods. Journal of Animal Science, v.43, n.4, p.835841, 1976. Disponível em: <http://jas.fass.org/cgi/reprint/ 43/4/835>. Acesso em: 07 jan. 2006

BORTOLUSSI, G. et al. Dietary nitrogen and phosphorus depletion in cattle and their effects on liveweight gain, blood metabolite concentrations and phosphorus kinetics. Journal of Agricultural Science, n.126, p.493-501, 1996. Disponível em: <http://journals.cambridge.org/action/ displayAbstract ? fromPage $=$ online $\&$ aid $=4802968>$. Acesso em: 07 jan. 2006. doi: 10.1017/S0021859600075584.

CONSTABLE, P.D. Hypertonic saline. Veterinary Clinics of North America: Food Animal Practice, v.15, n.3, p.559$585,1999$.

CONSTABLE, P.D. et al. Hypertonic saline is a negative inotropic agent in normovolumic dogs. American Journal of Phisiology, v.267, p.667-677, 1994. Disponível em: 
$<$ http://ajpheart.physiology.org/cgi/reprint/267/2/H667>. Acesso em: 07 jan. 2006.

DIBARTOLA, S. Anormalidades de fluidos, eletrólitos e equilíbrio ácido-básico na clínica de pequenos animais. 3.ed. São Paulo: Roca, 2007. 664p.

HALIBURTON, J.C.; MORGAN, S.E. Nonprotein nitrogeninduced ammonia toxicosis and ammoniated feed toxicity syndrome. Veterinary Clinics of North America: Food Animal Practice, v.5, n.2, p.237-249, 1989.

KANEKO, J.J. et al. Clinical biochemistry of domestic animals. 6.ed. San Diego: Academic, 2008. 928p.

KITAMURA, S.S. Intoxicação por amônia em bovinos e ratos: o desempenho renal na desintoxicação e o emprego de tratamentos alternativos. 2002. 92f. Dissertação (Mestrado em Clínica Veterinária) - Faculdade de Medicina Veterinária e Zootecnia, Universidade de São Paulo, SP

KITAMURA, S.S. et al. A model for ammonia poisoning in cattle. Veterinary and Human Toxicology, v.45, n.5, p.274$277,2003$.

LUTSGARTEN, J.A.; WENK, R.E. Simple, rapid, kinetic method for serum creatinine measurement. Clinical Chemistry, v.18, n.11, p.1419-1422, 1972. Disponível em: $<$ http://www.clinchem.org/cgi/reprint/18/11/1419>. Acesso em: 07 jan. 2006.

MARTIN Jr., D.W. et al. Harper's review of biochemistry. Los Altos: Lange Medical, 1981. 614p.

MINSON, D.J. Forage in ruminant nutrition. Queensland: Academic, 1990. 483p.

MORRIS, J.G.; PAYNE, E. Ammonia and urea toxicoses in sheep and their relation to dietary nitrogen intake. Journal
Agricultural Science, v.74, n.2, p.259-271, 1970. Disponível em: <http://journals.cambridge.org/action/ displayAbstract ? fromPage $=$ online $\&$ aid $=4568764>$. Acesso em: 07 jan. 2006. doi:10.1017/S0021859600022875.

OLIVEIRA, J.S. Utilização de cana+uréia na recria de bovinos. Coronel Pacheco: EMBRAPA - Centro Nacional de Pesquisa de gado de leite-CNPGL, 1985. 20p. (Circular técnica 23).

ORTOLANI, E.L.; ANTONELli, A.C. Acute ammonia poisoning in ruminants: a review. Current Topics in Toxicology, v.1, p.19-32, 2004.

ORTOLANI, E.L. et al. Ammonia toxicity from urea in a Brazilian dairy goat flock. Veterinary and Human Toxicology, v.42, n.2, p.87-89, 2000

ROEDER, B.L. et al. Acute effects of intravenously administered hypertonic saline solution on transruminal rehydration in dairy cows. American Journal Veterinary Research, v.58, n.5, p.549-554, 1997.

SAMPAIO, I.B.M. Estatística aplicada à experimentação animal. Belo Horizonte: UFMG, 1998. 221p.

SANTOS, N.V.M. Eficiência da desintoxicação de amônia em bovinos adaptados ou não com uréia dietética e submetidos a diferentes períodos de privação. 2005. 134f. Tese (Doutorado em Medicina Veterinária) - Faculdade de Medicina Veterinária e Zootecnia, Universidade de São Paulo, SP.

SIEGEL, S. Estatística não-paramétrica. São Paulo: Mcgraw-Hill, 1975. 350p.

TALKE, H.; SCHUBERT, G.E. Enzymatische harnstoffbestimmung in blut und serum in optishen test nach warburb. Klinische Wochenschrift, v.43, n.174, p.174$175,1965$. 